American Journal of Pharmacology and Toxicology 3 (1): 14-18, 2008

ISSN 1557-4962

(C) 2008 Science Publications

\title{
Life Extension by Anti-Aging Drugs: Hormetic Explanation?
}

\author{
A.M. Vaiserman \\ Laboratory of Mathematical Modelling of Aging Processes Institute of Gerontology, \\ Vyshgorodskaya st. 67, Kiev 04114, Ukraine
}

\begin{abstract}
A number of chemicals including antioxidants, vitamins and hormones are widely advertised as anti-aging drugs (geroprotectors). It is usually believed that life-extending capacity of geroprotectors may be attributed to their specific anti-aging mechanisms, even without solid scientific evidence. The analysis of the survival data gathered by both anti-aging and hormetic interventions demonstrates that many essential similarities exist between them. The evidence that life-extending effects of anti-aging drugs may be due to hormetic-like response is discussed in this review.
\end{abstract}

Key words: Anti-aging, geroprotectors, hormesis, longevity

\section{INTRODUCTION}

One of the most important problems in modern gerontology is the development of means to extend healthy lifespan. Recently, a number of nutrients and chemicals have been widely advertised as anti-aging drugs or supplements. Experimental studies have repeatedly shown the life-extending effect of such substances, often referred to as life-span prolonging drugs or geroprotectors ${ }^{[1]}$. The life-extending capacity was shown for a number of geroprotectors, including antioxidants, chelate agents and lathyrogens, succinate, adaptogens and herbs, neurotropic drugs, inhibitors of monoamine oxidase, glucocorticoids, dehydroepiandrosterone, sex and growth hormones, melatonin, pineal peptide preparations, protein inhibitors, antidiabetic biguanides, thymic hormones and peptides, immunomodulators and enterosorbents ${ }^{[2]}$, mimetics of superoxide dismutase and catalase ${ }^{[3]}$. The natural and synthetic dietary supplements and chemicals including antioxidants, vitamins and hormones are among the most popular products on the market, even without solid scientific evidence ${ }^{[4]}$. However, excessive intake of antioxidants or hormones is known to destroy delicate control mechanisms of homeostatic balance. It is therefore unlikely that they have a long-term beneficial impact ${ }^{[5]}$. Currently, there are no authentic anti-aging pharmaceuticals. However, this does not mean that they cannot exist ${ }^{[6]}$. One reason none has been found is that no assay has existed for identifying such drugs. Historically, the only accepted method of evaluating therapies that attempt to slow aging and the onset of age-related diseases has been lifespan studies. However, life extension per se hardly can be considered as strong evidence for a real antiaging action of the used compound. Life-extending effects were found for a number of obviously harmful substances like DDT and nuclear fallout. These effects are assumed to be caused by hormesis ${ }^{[7]}$. Hormesis is the concept that mild stimulation that is harmful at high doses can up-regulate physiological/biochemical processes in the body to produce an adaptive beneficial response at lower doses ${ }^{[8]}$. Chemical hormesis was observed in a wide range of taxonomic groups and involved agents representing highly diverse chemical classes $^{[9]}$. In experimental studies, chemical hormesis was repeatedly exhibited by feeding of antibiotics, herbicides, pesticides, insecticides, hydrocarbons, ethanol, solvents and several other classes of chemicals in various organisms, including plants, viruses, bacteria, fungi, insects, fish, birds, rodents and primates ${ }^{[10]}$. For gerontologists, most important are the survivalenhancing aspects of hormesis. Several mild stresses including irradiation, heat and cold shock, hypergravity, exercise, etc. have been reported to increase life $\operatorname{span}^{[11]}$. Longevity hormesis has been obtained by dietary supplementation with antibiotics, herbicides, pesticides, heavy metals and hydrocarbons ${ }^{[7]}$.

It is usually believed that the life-extending capacity of geroprotectors may be attributed to their specific anti-aging mechanisms. The hormeticallyinduced adaptive response, it seems, might be an alternative explanation for this. The evidence that lifeextending effect of the anti-aging drugs (geroprotectors) may be caused due to hormetic-like response is discussed in the next sections. 
Am. J. Pharm. \& Toxicol., 3 (1): 14-18, 2008

\section{LIFE EXTENSION PATTERNS BY ANTI-AGING DRUGS AND BY HORMETIC RESPONSE: FORMAL SIMILARITY OR EVIDENCE FOR COMMON MECHANISMS?}

George A. Sacher differentiated the life-extending effects of agents which act through what he termed a proper action from the hormetic actions arising from non-specific stimuli ${ }^{[12]}$. He defined a proper action as the specific biochemical role of an agent in reducing the accumulation of an aging lesion or protecting against age-dependent diseases. Sacher theorized that life extension due to hormesis is distinct from that due to proper actions by the form of age-specific logarithmic mortality curves and their Gompertz approximations (the exponential equation to approximate the probability of death as a function of age). For instance, he assumed that low doses of radiation result in a decrease of the Gompertz intercept (frailty), whereas caloric restriction (proper action, by Sacher) results in a decrease of the Gompertz slope (rate of senescence) ${ }^{[12]}$. At the present time, such distinctions seem irrelevant. For example, the effects of antioxidants as well as caloric restriction on the behavior of mortality curves in mice and rats do not indicate that these treatments alter the rates of aging ${ }^{[13]}$. Remarkably, many authors now recognize the life-extending and anti-aging actions of caloric restriction (most compelling example of life span extension by external manipulation) as an example of hormesis ${ }^{[14-16]}$ (though other authors do not share this view $\left.^{[10]}\right)$.

In 1962, Sacher and Trucco suggested that hormesis is a function of the state of the organism, not of the stimulus ${ }^{[17]}$. They postulated that hormesis could only be observed in animals in suboptimal environments or state of health and, therefore, hormesis would not result in an increase of maximal life span but would serve only to enable the animal to approach its potential longevity. All hormesis-induced mild stresses (excluding caloric restriction), were found to be able to extend the mean but not the maximal life $\operatorname{span}^{[6]}$. Similarly, antioxidant supplemented diets have been shown to have some degree of success in increasing mean, but not maximal, life $\operatorname{span}^{[18,19]}$. Current data indicate that antioxidants cannot prolong maximal life span and their beneficial impact on various age-related degenerative diseases may forecast an improvement in life span and enhance quality of life ${ }^{[20]}$. Sacher and Trucco also postulated that hormesis can be found only if control animals are short-lived ${ }^{[17]}$. Similarly, geroprotector efficiency depends on viability of the control population ${ }^{[21]}$. For example, for a relatively low life span in a population from which the control and experimental groups were formed, the geroprotective effect of melatonin was the most distinct; for a relatively high life span, the effect of the hormone was either not detected or appeared as a toxic reduction in life span (up to $10 \%$ ) in the experimental group $^{[22]}$.

Hormetic responses were generally observed to be of limited magnitude. In all experiments that displayed enhanced longevity, the average life span was enhanced by $10-30 \%{ }^{[4,6]}$. The magnitude of the life-extending effects of geroprotectors is consistent with that seen for hormetic responses. In most cases, the maximum magnitude does not exceed $20-30 \%{ }^{[23]}$.

The early-life administration was found to be preferable to obtain sufficient effects for both hormetic response and geroprotective intervention. Given the age-specific effects of dietary restriction on functional state, imposing dietary restriction late in life might not provide the same functional benefits as when applied at early ages ${ }^{[24]}$. Similarly, geroprotective interventions seem to be more effective if they are applied early rather than late in life $\mathrm{e}^{[9,23]}$. For instance, application of the geroprotector 2-ethyl-6-methyl-3-hydroxypyridine hydrochloride at the larval stage resulted in a 18-20\% increase in mean life span for both sexes in Drosophila melanogaster. The older the individuals were by the beginning of the experiment, the weaker was the effect of the geroprotector. The mean life span of females taken in the experiment at one day old was increased by $12 \%$; the influence at an older age than 20 days was ineffective ${ }^{[25]}$.

Anti-aging drugs: multi-functionality and complexity: The efficiency of substances that are believed to slow or prevent aging (anti-aging drugs) is usually attributed to specific geroprotective mechanisms. For example, according to the free radical theory of aging ${ }^{[26]}$, free radicals are involved in the production of changes in cellular metabolism that lead to a time-dependent functional decline in all living beings. Consequently, antioxidants and/or free radicals scavengers are believed to retard the aging process. However, a majority of potential geroprotectors are multi-functional. For example, vitamin $\mathrm{C}$ can act as an antioxidant, metal chelator, reducing agent or oxygen scavenger ${ }^{[27]}$. Aspirin may affect oxidant production, cytokine responses and block glycooxidation reactions ${ }^{[28]}$. Therefore, life-extending effect of geroprotector hardly can be attributed to a single antiaging mechanism.

Coexistence of beneficial and toxic effects: Mild forms of stress can promote mental and physical function whereas extreme stress is more likely to cause 
Am. J. Pharm. \& Toxicol., 3 (1): 14-18, 2008

mental anguish and physical ailments. In pharmacology, many chemicals are known to have opposite effects as a function of dosage (e.g., the antibiotics penicillin, erythromycin and streptomycin promote bacterial growth at low doses, contrary to effects at higher doses). Longevity hormesis results from exposure to agents which also possess toxicity ${ }^{[29]}$. Non-toxic or stimulatory effects promoting longevity do not preclude the coexistence of toxic effects, which simultaneously have a negative impact on survival. Hormesis and toxicity may be coexisting - the predominance of one or the other may vary not only with dose but also with species, gender, age, stage of life, disease or disability ${ }^{[11]}$.

The potential anti-aging supplements are essential nutrients necessary to healthy life ${ }^{[30]}$. However, similarly to the hormesis-inducing substances, these supplements show toxic adverse effects at excess levels (i.e., they demonstrate typical drug-dosage effects) ${ }^{[31,32]}$. The small daily levels of vitamins and minerals are both required and beneficial, while excessive dietary levels can lead to hypervitaminosis, tissue mineralization, or electrolyte imbalance. Vitamin A deficiency can have harmful effects while excessive vitamin A can damage the liver or cause birth defects; high doses of selenium can affect the brain and high doses of estrogens may increase the risk of breast cancer ${ }^{[33]}$. Aspirin has been found to be associated with a decreased risk of heart disease and other newly found health benefits. However, it produces allergy-like and toxic effects in many individuals ${ }^{[34]}$.

The most popular longevity-promoting nutrients, discussed in the consumer media and in medical literature, are antioxidants. According to the free radical theory of aging, first proposed by Harman ${ }^{[35]}$, free radical reactions contribute to degenerative aging changes and represent the basic cause of numerous human diseases, including heart disease, stroke, cancer, diabetes, cataracts, arthritis and neurodegenerative disorders. This theory implies that antioxidants (e.g. vitamin $\mathrm{A}$, vitamin $\mathrm{C}$ and vitamin $\mathrm{E}$ ), which prevent oxidative DNA damage, will slow the aging process ${ }^{[36]}$. Earlier observational studies have reported that antioxidants can improve health ${ }^{[37]}$. However, in recent years, compelling evidence has been accumulated showing the ineffectiveness and even dangers of antioxidants $^{[38,39]}$. Well-known antioxidants such as beta-carotene (vitamin $\mathrm{A})^{[40]}$, alpha-tocopherol (vitamin $\mathrm{E})^{[41]}$, ascorbic acid (vitamin $\left.\mathrm{C}\right)^{[42]}$ and melatonin ${ }^{[43]}$ may show pro-oxidant effects, depending on the dose and physiological radical defence and these reverse effects may increase oxidative damage ${ }^{[4,45]}$. Paradoxically, many well-established components of the heart-healthy lifestyle are prooxidant, including polyunsaturated fat, exercise and moderate alcohol consumption $^{[46]}$. Large randomized controlled trials are clearly showing significant antioxidant harm, particularly for beta carotene and vitamin E. The Bjelakovic et al., meta-analysis of studies published before October 2005, which pooled data from 68 studies involving more than 232,000 people, found that beta carotene, vitamin A and vitamin E, taken singly or combined with other antioxidant supplements, were associated with increased all-cause mortality ${ }^{[38]}$. When looked at separately, vitamin A increased death risk by 16 per cent, beta carotene by 7 per cent and vitamin $\mathrm{E}$ by 4 per cent. The results for vitamin $C$ were somewhat unclear, but by looking at the best quality trials there was a suggestion that it increased death risk by 6 per cent, either on its own or in combination with other supplements. There are many examples of essential trace elements exerting a U-shaped (hormetic-like) dose-response on physiological functioning, ranging from impairment at levels of deficient intake, to optimal function at intermediate levels and to toxicity at excessive intake levels ${ }^{[47]}$. The hormetic-like doseresponse has been observed for some anti-aging compounds including antioxidants. For example, when the exogenous antioxidant (superoxide dismutase mimetic drug Euk-8) was fed to adult SOD-deficient Drosophila melanogaster, female lifespan was significantly increased at $0,1 \mathrm{mM}$ and $1 \mathrm{mM}$, but decreased dramatically when the dose was increased to $10 \mathrm{mM}^{[31]}$. U-shaped association between vitamin $\mathrm{C}$ intake and death from stroke was found in 34,492 postmenopausal women ${ }^{[48]}$. The phytoestrogens, which are believed to have estrogen-like benefits and also give both antioxidant protection and protective effect against breast cancer due to aromatase inhibition, were aromatase inhibitors at low concentrations $(<1 \mu \mathrm{M})$ but estrogenic at higher concentrations $(\geq 1 \mu \mathrm{M})$, resulting in U-shaped dose-response curves[ ${ }^{32]}$.

Therefore, effects of ant-aging drugs seem to be rather unspecific and have some similarity with hormetic effects. It can be hypothesized that these drugs could cause benefits because they were applied within the optimal hormetic response zone for these substances.

\section{CONCLUSIONS}

As can be seen from the discussion above, striking similarities between the geroprotetive and hormetic lifeextending studies do exist. Therefore, the question arises: are there common mechanisms underlying lifeextending effects of anti-aging drugs and hormetic response? 
Aging can be characterized by a decrease in adaptive abilities due to extensive damage of maintenance and repair pathways. In contrast, hormesis-inducing stresses can stimulate an organism's maintenance and repair pathways which increase adaptive abilities. The efficiency of anti-aging drugs is usually attributed to specific (molecular, cellular etc.) geroprotective mechanisms. However, rather than changes in individual cells, tissues, or organs, aging may be more a function of the deterioration of integrative mechanisms, for instance, in the central nervous system ${ }^{[49]}$. The hormetic response is also suggested to be regulated by integrative mechanisms ${ }^{[11]}$. Conceptually, hormesis may be more efficient to promote health than uptake of supplements because it could induce the body's own capabilities in good balance with numerous interacting metabolic processes to combat more severe stresses ${ }^{[5]}$. Therefore, the search for new anti-aging treatments will likely be more effective by utilizing the hormetic response at the whole organism level.

\section{REFERENCES}

1. Emanuel, L.M. and L.K. Obukhova, 1978. Types of experimental delay in aging patterns. Exp. Gerontol., 13: 25-29.

2. Anisimov, VN., 2001. Life span extension and cancer risk: Myths and reality. Exp. Gerontol., 36: 1101-1136.

3. Melov, S., J. Ravenscroft, S. Malik, M.S. Gill, D.W. Walker, P.E. Clayton, D.C. Wallace, B. Malfroy, S.R. Doctrow and G.J. Lithgow, 2000. Extension of life-span with superoxide dismutase/catalase mimetics. Science, 289: 1567-1569.

4. Olshansky, S.J., L. Hayflick and B.A. Carnes, 2002. No truth to the fountain of youth. Sci. Am., 286: 92-95.

5. Goto, S., 2004. Hormesis and intervention of aging: An emerging paradigm in gerontology. Geriatrics and Gerontol. Int., 4: S79-S80.

6. Spindler, S.R., 2003. Caloric Restriction, Longevity and the Search for Authentic AntiAging Drugs, In: Anti-Aging Therapy for Plastic Surgery (eds B. Kinney and J. Carraway). Quality Medical Publishing, Inc., St. Louis.

7. Neafsey, P.J., 1990. Longevity hormesis. A review. Mech. Ageing Dev., 51: 1-31.

8. Calabrese, E.J. and L.A. Baldwin, 2002. Defining hormesis. Hum. Exp. Toxicol., 21: 91-97.
9. Calabrese, E.J. and L.A. Baldwin, 1998. Hormesis as a biological hypothesis. Environ. Health Perspect., 106: 357-362.

10. Calabrese, E.J. and R. Blain, 2005. The occurrence of hormetic dose responses in the toxicological literature, the hormesis database: An overview. Toxicol. Applied Pharmacol., 202: 289-301.

11. Minois, N., 2000. Longevity and aging: Beneficial effects of exposure to mild stress. Biogerontol., 1: 15-29.

12. Sacher, G.A., 1977. Life Table Modification and Life Prolongation. In: Handbook of the Biology of Aging. Finch C.E. and L. Hayflick (Eds.). pp: 582-638. Van Nostrand-Reinhold, New York.

13. Doubal, S. and P. Klemera,1999. The effect of antioxidants and dietary restriction on mortality curves. Age, 22: 101-105.

14. Parsons, P.A., 2000. Caloric restriction, metabolic efficiency and hormesis. Hum. Exp. Toxicol., 19: 345-347.

15. Turturro, A., B.S. Hass and R.W. Hart, 2000. Does caloric restriction induce hormesis? Hum. Exp. Toxicol., 6: 320-329.

16. Masoro, E., 1998. Hormesis and the anti-aging action of caloric restriction. Exp. Gerontol., 33: 61-66.

17. Sacher, G.A. and E. Trucco, 1962. A Theory of the Improved Performance and Survival Produced by Small Doses of Radiations and Other Poisons. In: Biological Aspects of Aging. Shock, N.W., (Ed.). pp: 244-251. Columbia University Press, New York.

18. Weber, H. and J. Miquel, 1986. Antioxidant Supplementation and Longevity. In: Nutritional Aspects of Aging. Chen, L.H., (Ed.). pp: 2-49.CRC Press, Boca Raton, Florida.

19. Bayne, A.C. and R.S. Sohal, 2002. Effects of superoxide dismutase/catalase mimetics on lifespan and oxidative stress resistance in the housefly Musca domestica. Free Radical Biol. Med., 32: 1229-1234.

20. Bonnefoy, M., J. Drai and T. Kostka, 2002. Antioxidants to slow aging, facts and perspectives. Presse Med., 31: 1174-1184.

21. Izmaylov, D.M. and L.K. Obukhova, 1996. Geroprotector efficiency depends on viability of control population: life span investigation in D. melanogaster. Mech. Ageing Dev., 91: 155-164.

22. Izmaylov, D.M. and L.K. Obukhova, 1999. Geroprotector effectiveness of melatonin: investigation of lifespan of Drosophila melanogaster. Mech. Ageing Dev., 106: 233-240. 
23. Frolkis, V.V. and K.K. Muradian, 1991. Experimental Life Prolongation. CRC Press, New York.

24. Burger, J.M., D.S. Hwangbo, V. Corby-Harris and D.E. Promislow, 2007. The functional costs and benefits of dietary restriction in Drosophila. Aging Cell, 6: 63-71.

25. Nakaidze, N.Sh., L.K. Obukhova, L.D. Smirnov and A.P. Akifyev, 1978. Influence of the geroprotector 2-ethyl-6-methyl-3-hydroxypyridine hydrochloride on the lifetime of Drosophila melanogaster. Biol. Bull. Acad. Sci. USSR, 5: 505-508.

26. Ashok, B.T. and R. Ali, 1999. The aging paradox: free radical theory of aging. Exp. Gerontol., 34: 293-303.

27. Aliste, A.J. and N.L. Del Mastro, 2004. Ascorbic acid as radiation protector on polysaccharides used in food industry. Colloids and Surfaces A: Physicochemical and Engineering Aspects, 249: 131-133.

28. Phillips, T. and Ch. Leeuwenburgh, 2004. Lifelong aspirin supplementation as a means to extending life span. Rejuvenation Research, 7: 243 -252.

29. Neafsey, P.J., H. Boxenbaum, D.A. Ciraulo and D.J. Fournier, 1989. A Gompertz age-specific mortality rate model of aging, hormesis and toxicity: dose-response studies. Drug Metab. Rev., 20: 111-150.

30. Eaton, D.L. and C.D. Klaassen, 2001. Principles of Toxicology. In: Casarett and Doull's Toxicology: The Basic Science of Poisons, Chapter 2. Klaassen, C.D., (Ed.). 6th Edn., pp: 13-33. McGraw-Hill, New York.

31. Magwere, T., M. West, K. Riyahi, M.P. Murphy, R.A. Smith and L. Partridge, 2006. The effects of exogenous antioxidants on lifespan and oxidative stress resistance in Drosophila melanogaster. Mech. Ageing Dev., 127: 356-370.

32. Almstrup, K., M.F. Fernandez, J.H. Petersen, N. Olea, N.E. Skakkebaek and H. Leffers, 2002. Dual effects of phytoestrogens result in U-shaped doseresponse curves. Environ. Health Perspect. 110: 743-748.

33. Hayes, D.P., 2007. Nutritional hormesis. Eur. J. Clin. Nutr., 61: 147-159.

34. Feinman, S.E., 1993. Beneficial and Toxic Effects of Aspirin (Pharmacology and Toxicology). CRC Press, Boca Raton, Florida.

35. Harman, D., 1956. Aging: a theory based on free radical and radiation chemistry. J. Gerontol., 11: 298-300.
36. Harman, D. 1994. Free radical theory of aging. Increasing the functional life span. Ann. N.Y. Acad. Sci., 717: 1-15.

37. Diplock, A.T., 1994. Antioxidants and disease prevention. Mol. Aspects Med., 15: 293-376.

38. Bjelakovic, G., D. Nikolova, L.L. Gluud, R.G. Simonetti and Ch. Gluud, 2007. Mortality in randomized trials of antioxidant supplements for primary and secondary prevention: Systematic review and meta-analysis. JAMA, 297: 842-857.

39. Herbert, K.E., S. Fletcher, D. Chauhan, A. Ladapo, J. Nirwan, S. Munson and P. Mistry, 2006. Dietary supplementation with different vitamin $\mathrm{C}$ doses: No effect on oxidative DNA damage in healthy people. Eur. J. Nutr., 45: 97-104.

40. Yeh, S.L. and M.L. Hu, 2000. Antioxidant and prooxidant effects of lycopene in comparison with beta-carotene on oxidant-induced damage in Hs68 cells. J. Nutr. Biochem., 11: 548-554.

41. Maiorino, M., A. Zamburlim, A. Roveri and F. Ursini, 1993. Prooxidant role of vitamin $\mathrm{E}$ in copper induced lipid peroxidation. FEBS Lett., 330: 174-176.

42. Duarte, T.L. and J. Lunec, 2005. When is an antioxidant not an antioxidant? A review of novel actions and reactions of vitamin C. Free Radic. Res., 39: 671-686.

43. Osseni, R.A., P. Rat, A. Bogdan, J.M. Wamet and Y. Touitou, 2000. Evidence of prooxidant and antioxidant action of melatonin on human liver cell line HepG2. Life Sci., 68: 387-399.

44. Halliwell, B., 2000. The antioxidant paradox. The Lancet, 355:1179-1180.

45. Bakan, V., I. Demirtas and H. Dulger, 2004. The antioxidant paradox or antioxidant damage. Pediatric Surgery International, 20: 903.

46. Williams, K.J. and E.A. Fisher, 2005. Oxidation, lipoproteins and atherosclerosis: which is wrong, the antioxidants or the theory? Current Opinion in Clinical Nutrition and Metabolic Care, 8: 139-146.

47. Mertz, W., 1981. The essential trace elements. Science, 213, 580-583.

48. Yochum, L.A., A.R. Folsom and L.H. Kushi, 2000. Intake of antioxidant vitamins and risk of death from stroke in postmenopausal women. Am. J. Clin. Nutr., 72: 476-483.

49. Shock, N.W., 1977. Systems Integration. In: Handbook of the Biology of Aging, Finch, C.E. and L. Hayflick (Eds.). pp: 639-665. Van Nostrand Reeinhold, New York. 